\title{
Seletividade de Herbicidas Registrados para a Cultura do MILHO A AdULTOS DE Trichogramma pretiosum (HYMENOPTERA: TRICHOGRAMMATIDAE) ${ }^{1}$
}

\author{
Selectivity of Herbicides Registered on Corn to Trichogramma pretiosum (Hymenoptera: \\ Trichogrammatidae)
}

\author{
STEFANELLO JÚNIOR, G.J. ${ }^{2}$, GRÜTZMACHER, A.D. ${ }^{3}$, GRÜTZMACHER, D.D. ${ }^{4}$, LIMA, C.A.B. ${ }^{5}$, \\ DALMOZO, D.O. ${ }^{6}$ e PASCHOAL, M.D.F. ${ }^{7}$
}

\begin{abstract}
RESUMO - A seletividade de 24 herbicidas registrados para a cultura do milho foi avaliada a Trichogramma pretiosum em condições de laboratório (temperatura de $25 \pm 1{ }^{\circ} \mathrm{C}$, umidade relativa de $70 \pm 10 \%$, fotofase de 14 horas e luminosidade de 500 lux). Adultos de T. pretiosum foram colocados em contato com uma película seca dos herbicidas pulverizados sobre placas de vidro e avaliou-se a capacidade de parasitismo das fêmeas. A redução na capacidade de parasitismo dos tratamentos foi comparada com a da testemunha (água destilada) e utilizada para classificar os herbicidas em 1, inócuo (<30\%); 2, levemente nocivo (30-79\%); 3, moderadamente nocivo (80-99\%); e 4, nocivo (>99\%). Os herbicidas Callisto, Equip Plus, Extrazin SC, Primóleo, Provence 750 WG e Siptran 500 SC são inócuos (classe 1); Agrisato 480 SL, Gesaprim GrDA, Glifos, Glyphosate Nortox, Gliz 480 SL, Polaris, Primatop SC, Sanson 40 SC, Trop e Zapp Qi, levemente nocivos (classe 2); Finale, Herbadox, Poast, Roundup Original, Roundup Transorb e Roundup WG, moderadamente nocivos (classe 3); e Gramoxone 200 e Primestra Gold, nocivos (classe 4) aos adultos de T. pretiosum, nas dosagens utilizadas. Os herbicidas nocivos (classes 2, 3 e 4) deverão passar para as etapas seguintes, que envolverão testes sobre as fases imaturas do parasitóide em condições de laboratório e adultos a campo.
\end{abstract}

Palavras-chave: controle químico, efeitos colaterais, inimigo natural, parasitóide de ovos, Zea mays.

\begin{abstract}
The selectivity of 24 herbicides registered on corn to Trichogramma pretiosum (Hymenoptera: Trichogrammatidae) was assessed under laboratory conditions $\left(25 \pm 1{ }^{\circ} \mathrm{C}\right.$ temperature, $70 \pm 10 \%$ relative humidity, 144 photophase and brightness 500 lux). The adult parasitoids were submitted to a dry film of the herbicides applied on glass plates and the parasitism capacity of the females was evaluated. Reduced parasitism capacity in the treatments was compared with the negative control (distilled water) and used to classify the herbicides into four categories: 1, harmless (< 30\%); 2, slightly harmful (30-79\%); 3, moderately harmful (80-99\%) and 4, harmful (> 99\%). The herbicides Callisto, Equip Plus, Extrazin SC, Primóleo, Provence 750 WG and Siptran 500 SC were found to be harmless (class 1); Agrisato 480 SL, Gesaprim GrDA, Glifos, Glyphosate Nortox, Gliz 480 SL, Polaris, Primatop SC, Sanson 40 SC, Trop and Zapp Qi, slightly harmful (class 2); Finale, Herbadox, Poast, Roundup Original, Roundup Transorb and Roundup WG,
\end{abstract}

1 Recebido para publicação em 11.7.2007 e na forma revisada em 20.3.2008.

Parte da dissertação do primeiro autor apresentada ao Programa de Pós-Graduação em Fitossanidade da Universidade Federal de Pelotas, Capão do Leão-RS, Brasil.

2 Engo-Agr ${ }^{0}$, aluno do Programa de Pós-Graduação em Fitossanidade da UFPel, Caixa Postal 354, 96010-900, Capão do Leão, RS,

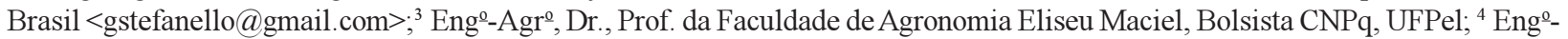
Agr ${ }^{\circ}$, Dr., Bolsista PRODOC-CAPES, Programa de Pós-Graduação em Fitossanidade, UFPel; ${ }^{5}$ Bióloga, aluna do Programa de PósGraduação em Fitossanidade, UFPel; ${ }^{6}$ Acadêmico de Agronomia, bolsista PIBIC CNPq, UFPel; ${ }^{7}$ Acadêmico de Agronomia, Bolsista PET, UFPel 
moderately harmful (class 3); Gramoxone 200 and Primestra Goldwere found to be harmful (class 4) to the adults of T. pretiosum at the dosages used. The harmful herbicides (class 2, 3 and 4) must undergo the next test stages, involving tests at the immature phases of the parasitoid under laboratory conditions and adult parasitoids under field conditions.

Keywords: chemical control, side effects, natural enemy, egg parasitoid, Zea mays.

\section{INTRODUÇÃO}

A cultura do milho no Brasil ocupa uma área aproximada de 11,5 milhões de hectares, dos quais 8,5 e 2,9 milhões são destinados à primeira e à segunda safra, respectivamente, com produtividade média de $3.351 \mathrm{~kg} \mathrm{ha}^{-1} \mathrm{e}$ produção de mais de 42 milhões de toneladas por ano (IBGE, 2007). No entanto, uma parcela da redução da produtividade dessa cultura se deve às altas populações de insetos e de plantas daninhas. Dentre os insetos-praga, destacamse a lagarta-do-cartucho Spodoptera frugiperda (Lepidoptera: Noctuidae) e a lagarta-da-espiga Helicoverpa zea (Lepidoptera: Noctuidae), as quais causam danos diretos e indiretos e perdas na produção.

No grupo dos inimigos naturais de $S$. frugiperda e $H$. zea destacam-se os himenópteros do gênero Trichogramma (Sá \& Parra, 1993; Cruz, 1995), que apresentam como principal vantagem o controle desses insetos-praga ainda na fase de ovo, ao parasitá-los, ou seja, antes de causarem qualquer dano à cultura. Assim, considerando a ampla representatividade no grupo dos parasitóides, em que a ordem Hymenoptera perfaz cerca de $80 \%$ destes (Viñuela, 2002), e a distribuição geográfica mundial, o grupo de trabalho da "International Organization for Biological and Integrated Control of Noxious Animals and Plants (IOBC), West Palaearctic Regional Section (WPRS)", escolheu Trichogramma como um gênero-padrão de parasitóides para realização de testes de seletividade para registro de novos agrotóxicos.

No entanto, espécies do gênero Trichogramma são fototrópicas positivas e apresentam máxima atividade de oviposição durante o dia (Cruz, 1995), estando, por isso, mais expostas aos efeitos tóxicos da aplicação de agrotóxicos não-seletivos. Dessa forma, adultos de Trichogramma podem ser atingidos ou entrar em contato com o herbicida durante o manejo de plantas daninhas, seja na dessecação para o plantio-direto, na pulverização convencional ou em pós-emergência dirigida. Ademais, a mistura de inseticidas e herbicidas para o manejo de insetos e plantas daninhas, respectivamente (Silva et al., 2007), ou a utilização de herbicidas dessecantes, visando a antecipação da colheita (Magalhães et al., 2005), também podem afetar negativamente a população desse parasitóide na cultura do milho.

A seletividade de herbicidas aos adultos de Trichogramma pretiosum foi avaliada por Giolo et al. (2005a), aos quais verificaram diferenças na toxicidade ao parasitóide quando utilizaram distintos produtos comerciais com base no mesmo ingrediente ativo. Nesse sentido, os testes de seletividade aos organismos não-alvo devem ser conduzidos com as formulações comerciais dos agrotóxicos, uma vez que um mesmo ingrediente ativo pode estar presente em diferentes formulações comerciais e concentrações e, assim, causar impacto diferenciado sobre os organismos benéficos (Hassan et al., 2000).

No Brasil, estudos de seletividade de agrotóxicos a Trichogramma foram conduzidos predominantemente para frutíferas e olerícolas (Castelo Branco \& França, 1995; Carvalho et al., 2003; Giolo et al., 2005b; Manzoni et al., 2006a, 2007; Morandi Filho et al., 2006; Moura et al., 2006), e poucos foram os trabalhos em culturas anuais (Hohmann, 1993; Cañete, 2005), sobretudo na cultura do milho, em que somente alguns inseticidas foram avaliados (Cruz, 1995; Pratissoli et al., 2004). Assim, na maioria dessas culturas, poucos trabalhos foram realizados visando avaliar a seletividade de herbicidas a inimigos naturais.

Dessa maneira, o presente trabalho objetivou avaliar a seletividade de herbicidas registrados para a cultura do milho aos adultos de T. pretiosum em condições de laboratório, 
utilizando a metodologia preconizada pelo grupo de trabalho da IOBC/WPRS.

\section{MATERIAL E MÉTODOS}

Os trabalhos foram desenvolvidos nos Laboratórios de Controle Biológico e de Pesticidas do Departamento do Fitossanidade da Faculdade de Agronomia "Eliseu Maciel", Universidade Federal de Pelotas, RS. Os parasitóides $T$. pretiosum foram obtidos da criação mantida em laboratório e multiplicados em ovos do hospedeiro Anagasta kuehniella (Lepidoptera: Pyralidae). Os testes de seletividade foram conduzidos em laboratório (temperatura de $25 \pm 1{ }^{\circ} \mathrm{C}$, umidade relativa de $70 \pm 10 \%$; fotofase de 14 horas e luminosidade de 500 lux), expondo-se adultos do parasitóide (estádio mais sensível) aos resíduos secos dos herbicidas (Tabela 1), adaptando-se as metodologias laboratoriais padronizadas de Trichogramma cacoeciae (Hassan et al., 2000; Hassan \& Abdelgader, 2001) à espécie T. pretiosum (Giolo et al., 2005b).

A seletividade dos herbicidas ao inimigo natural foi avaliada utilizando-se a máxima dosagem registrada para a cultura do milho (Tabela 1), totalizando 24 herbicidas testados em seis bioensaios. Os herbicidas foram diluídos em água destilada, considerando um volume de calda de $200 \mathrm{~L} \mathrm{ha}^{-1}$, e as aplicações foram feitas sobre placas de vidro $(0,2 \mathrm{~cm}$ de espessura x $13 \mathrm{~cm} \times 13 \mathrm{~cm}$ ) com deposição de calda de 1,75 $\pm 0,25 \mathrm{mg} \mathrm{cm}^{-2}$, aferida por pesagem em balança eletrônica de precisão, para posteriormente serem utilizadas na confecção das gaiolas de exposição, conforme Hassan \& Abdelgader (2001). Para a testemunha negativa foi utilizada água destilada e, para a testemunha positiva, o inseticida Lorsban $480 \mathrm{BR}$ (Tabela 1), por ser reconhecidamente nocivo (classe 4) a Trichogramma pela IOBC e registrado para a cultura do milho.

Os adultos de $T$. pretiosum, com aproximadamente 24 horas de idade, foram introduzidos nas gaiolas de exposição através de tubos de vidro de $12 \mathrm{~cm}$ de comprimento $\mathrm{x} 2 \mathrm{~cm}$ de diâmetro na extremidade $x 0,7 \mathrm{~cm}$ na extremidade oposta, denominados tubos de emergência, fazendo-se a conexão desse tubo na lateral da gaiola, em conformidade com Hassan \& Abdelgader (2001). Seis horas após a desconexão dos tubos de emergência, cartões contendo três círculos de $1 \mathrm{~cm}$ de diâmetro com $450 \pm 50$ ovos de A. kuehniella inviabilizados e alimento (solução composta por $200 \mathrm{~g}$ de mel, $3 \mathrm{~g}$ de gelatina em pó sem sabor e $100 \mathrm{~mL}$ de água) foram oferecidos às 24 (três cartões), 48 (dois cartões) e 96 (um cartão) horas após pulverização para serem parasitados por T. pretiosum, totalizando 18 círculos, com aproximadamente 8.100 ovos, por gaiola. A avaliação da capacidade de parasitismo foi mantida por 144 horas (seis dias). Posteriormente, as gaiolas foram desmontadas e os cartões foram preservados em placas de Petri $(9,0 \times 1,5 \mathrm{~cm})$, os quais permaneceram nas mesmas condições experimentais por mais três dias, para que todos os ovos parasitados se tornassem escuros e, assim, possibilitassem a contagem.

Foram utilizadas quatro repetições para cada tratamento, sendo cada gaiola considerada uma unidade experimental no delineamento inteiramente casualizado. Aos resultados foram atribuídas as classes propostas pela IOBC/WPRS, com base na redução do parasitismo dos adultos de $T$. pretiosum, quando comparadas com a testemunha negativa. Dessa forma, os herbicidas foram classificados em 1 - inócuo $(<30 \%)$; 2 - levemente nocivo (30$79 \%$ ); 3 - moderadamente nocivo (80-99\%); e 4 - nocivo (> 99\%). A classificação foi realizada em função do produto comercial, de acordo com Hassan et al. (2000), pois um mesmo ingrediente ativo poderá estar em distintas formulações comerciais e, assim, ocasionar impacto diferenciado sobre o inseto em teste.

Análises estatísticas foram realizadas com auxílio do programa estatístico SAS - Statistical Analysis System (SAS, 2002). Os resultados obtidos, relativos ao número de ovos parasitados por fêmea, foram testados quanto à normalidade pelo teste de Shapiro-Wilk, utilizando o procedimento UNIVARIATE. Não atendida essa pressuposição, foi realizada análise nãoparamétrica pelo procedimento NPAR1WAY (Kruskal-Wallis) e, após comprovação da existência de diferença entre tratamentos, os dados foram transformados pelo procedimento RANK. A comparação de médias foi obtida pelo teste Bonferroni-Dunn $t$, utilizando-se o procedimento GLM em nível de 5\% de probabilidade de erro. Em caso de normalidade dos dados, as médias foram comparadas pelo teste 
Tabela 1 - Herbicidas avaliados nos testes de seletividade a adultos de Trichogramma pretiosum, utilizando dosagem máxima do produto comercial registrada para a cultura do milho. Pelotas-RS. 2005/2006

\begin{tabular}{|c|c|c|c|c|c|}
\hline Produto comercial & Ingrediente ativo & Grupo químico & $\mathrm{DC}^{1}$ & C.i.a. ${ }^{2}$ & C.p.c. ${ }^{3}$ \\
\hline Agrisato $^{\circledR} 480 \mathrm{SL}$ & glyphosate - sal de isopropilamina & Glicina substituída & 6,00 & $1,08^{4}$ & 3,00 \\
\hline Callisto $^{\circledR}$ & mesotriona & Tricetona & 0,40 & 0,10 & 0,20 \\
\hline Equip Plus $^{\circledR}$ & foramsulfuron + iodosulfuron-metílico & Sulfoniluréia + Sulfoniluréia & 0,15 & $0,02+0,002$ & 0,08 \\
\hline Extrazin $^{\circledR} \mathrm{SC}$ & atrazine + simazine & Triazina + Triazina & 6,80 & $0,85+0,85$ & 3,40 \\
\hline Finale $^{\circledR}$ & glufosinato - sal de amônio & Homoalanina substituída & 1,50 & 0,15 & 0,75 \\
\hline Gesaprim $^{\circledR}$ GrDA & atrazine & Triazina & 3,50 & 1,54 & 1,75 \\
\hline Glifos $^{\circledR}$ & glyphosate - sal de isopropilamina & Glicina substituída & 2,00 & $0,36^{4}$ & 1,00 \\
\hline Glyphosate Nortox $^{(\mathbb{B}}$ & glyphosate - sal de isopropilamina & Glicina substituída & 6,00 & $1,08^{4}$ & 3,00 \\
\hline $\mathrm{Gliz}^{(\mathbb{B}} 480 \mathrm{SL}$ & glyphosate - sal de isopropilamina & Glicina substituída & 6,00 & $1,08^{4}$ & 3,00 \\
\hline Gramoxone $^{(\mathbb{B}} 200$ & dicloreto de paraquat & Bipiridilo & 3,00 & 0,30 & 1,50 \\
\hline Herbadox $^{\circledR}$ & pendimethalin & Dinitroanilina & 3,50 & 0,88 & 1,75 \\
\hline Poast $^{\circledR}$ & setoxidim & Oxima ciclohexanodiona & 1,25 & 0,12 & 0,63 \\
\hline Polaris $^{(3)}$ & glyphosate - sal de isopropilamina & Glicina subsituída & 5,00 & $0,90^{4}$ & 2,50 \\
\hline Primatop $^{\mathbb{Q}} \mathrm{SC}$ & atrazine + simazine & Triazina + Triazina & 8,00 & $1,00+1,00$ & 4,00 \\
\hline Primestra Gold $^{\mathbb{B}}$ & atrazine + S-metolachlor & Triazina + Cloroacetanilida & 4,50 & $0,83+0,65$ & 2,25 \\
\hline Primóleo $^{\circledR}$ & atrazine & Triazina & 6,00 & 1,20 & 3,00 \\
\hline Provence $^{\circledR} 750 \mathrm{WG}$ & isoxaflutole & Isoxazol & 0,08 & 0,03 & 0,04 \\
\hline Roundup Original $^{(8)}$ & glyphosate - sal de isopropilamina & Glicina substituída & 6,00 & $1,08^{4}$ & 3,00 \\
\hline Roundup Transorb $^{B}$ & glyphosate - sal de isopropilamina & Glicina substituída & 4,50 & $1,08^{4}$ & 2,25 \\
\hline Roundup $^{(B)}$ WG & glyphosate - sal de amônio & Glicina substituída & 3,5 & $1,26^{4}$ & 1,75 \\
\hline Sanson $^{B} 40 \mathrm{SC}$ & Nicosulfuron & Sulfoniluréia & 1,50 & 0,03 & 0,75 \\
\hline $\operatorname{Siptran}^{B} 500 \mathrm{SC}$ & Atrazine & Triazina & 6,20 & 1,55 & 3,10 \\
\hline Trop $^{(B)}$ & glyphosate - sal de isopropilamina & Glicina substituída & 6,00 & $1,08^{4}$ & 3,00 \\
\hline Zapp $\mathrm{Qi}^{(\mathbb{R})}$ & glyphosate - sal de potássio & Glicina substituída & 4,20 & $1,05^{4}$ & 2,10 \\
\hline Lorsban $^{\circledR} 480 \mathrm{BR}^{5}$ & Clorpirifós & Organofosforado & 1,00 & 0,24 & 0,50 \\
\hline
\end{tabular}

${ }^{1 /} \mathrm{DC}=$ dosagem de campo $\left(\mathrm{L} \mathrm{ha}^{-1}\right.$ do produto comercial $)$ considerando um volume de calda de $200 \mathrm{~L} \mathrm{ha}^{-1}$

2/ C.i.a. = concentração $(\%)$ do ingrediente ativo na calda utilizada nos bioensaios.

${ }^{3 /}$ C.p.c. $=$ concentração $(\%)$ do produto comercial na calda usada nos bioensaios.

${ }^{4 /}$ Concentração (\%) do equivalente ácido na calda utilizada nos bioensaios.

${ }^{5 /}$ Testemunha positiva - inseticida reconhecidamente nocivo pela IOBC/WPRS, usado como padrão de toxicidade.

de Tukey (dados balanceados) e Tukey-Kramer (dados não balanceados), empregando o procedimento GLM em nivel de $5 \%$ de probabilidade de erro.

\section{RESULTADOS E DISCUSSÃO}

Foram observadas diferenças significativas entre os tratamentos nos seis bioensaios realizados (Tabela 2), verificando-se parasitismo, que variou de 0,01 a 28,88 ovos por fêmea de T. pretiosum. Embora o grupo de trabalho da IOBC/WPRS não utilize os resultados da análise estatística para diferenciação dos agrotóxicos quanto à toxicidade a Trichogramma, observa-se que há certa correlação entre esses dados. Alguns herbicidas mais inócuos - embora nem sempre aqueles classificados como classe 1 - não diferiram da testemunha, assim como os herbicidas mais nocivos não diferiram de Lorsban ${ }^{\circledR} 480$ BR, inseticida de alta toxicidade utilizado na testemunha positiva, ou apresentaram resultados muito próximos dos dele.

O número de fêmeas adultas de T. pretiosum no interior das gaiolas de exposição variou de 94,36 a 195,80 (Tabela 2). Segundo Zhang \& Hassan (2000), valores entre 55 e 150 fêmeas de $T$. cacoeciae no interior das gaiolas de exposição não interferiram nos resultados obtidos pelos autores. Por outro lado, Hassan (1998) afirma que o número de fêmeas no interior das gaiolas pode variar de 200 a 400 para a metodologia proposta pela IOBC/ 
Tabela 2 - Número médio de fêmeas por gaiola e efeito de herbicidas utilizados na cultura do milho sobre o número ( \pm EP) de ovos parasitados por fêmea, redução (\%) na capacidade de parasitismo de adultos de Trichogramma pretiosum e classificação de toxicidade (temperatura de $25 \pm 1{ }^{\circ} \mathrm{C}$, umidade relativa de $70 \pm 10 \%$, fotofase de 14 horas e luminosidade de 500 lux). Pelotas-RS $2005 / 2006$

\begin{tabular}{|c|c|c|c|c|c|c|}
\hline Produto comercial/ingrediente ativo & $\mathrm{DC}^{1}$ & C.i.a..$^{\prime}$ & Fêmea por gaiola & Ovo parasitado por fêmea ${ }^{3 /}$ & $\mathrm{RP}^{4 /}$ & $\begin{array}{l}\text { Classes } \\
\text { IOBC }^{5}\end{array}$ \\
\hline \multicolumn{7}{|c|}{ Bioensaio I } \\
\hline Água destilada / testemunha negativa & - & - & 156,73 & $25,73 \mathrm{a}$ & - & - \\
\hline Polaris $^{(B} /$ glyphosate & 5,00 & $0,90^{6}$ & 120,70 & $6,35 \mathrm{ab}$ & 75,33 & 2 \\
\hline Trop $^{\beta} /$ glyphosate & 6,00 & $1,08^{6}$ & 159,32 & $5,16 \mathrm{~b}$ & 79,97 & 2 \\
\hline Roundup Original $^{\mathbb{R}} /$ glyphosate & 6,00 & $1,08^{6}$ & 195,80 & $4,84 \mathrm{bc}$ & 81,19 & 3 \\
\hline Roundup Transorb ${ }^{\mathbb{B}}$ / glyphosate & 4,50 & $1,08^{6}$ & 144,17 & $3,85 \mathrm{bc}$ & 85,03 & 3 \\
\hline Lorsban $^{8 / 8} 480 \mathrm{BR} /$ clorpirifós $^{7}$ & 1,00 & 0,24 & 180,61 & $0,00 \mathrm{c}$ & 100,00 & 4 \\
\hline \multicolumn{7}{|c|}{ Bioensaio II } \\
\hline Água destilada / testemunha negativa & - & - & 152,02 & $32,64 \pm 2,56 \mathrm{a}$ & - & - \\
\hline Glifos $^{B} /$ glyphosate & 2,00 & $0,36^{6}$ & 160,76 & $11,76 \pm 0,87 \mathrm{~b}$ & 63,97 & 2 \\
\hline $\mathrm{Gliz}^{\mathbb{B}} 480 \mathrm{SL} /$ glyphosate & 6,00 & $1,08^{6}$ & 153,49 & $9,09 \pm 1,36 \mathrm{~b}$ & 72,15 & 2 \\
\hline Glyphosate Nortox $^{\mathbb{R}} /$ glyphosate & 6,00 & $1,08^{6}$ & 162,76 & $8,62 \pm 1,45 b$ & 73,58 & 2 \\
\hline Agrisato $^{(8)} 480 \mathrm{SL} /$ glyphosate & 6,00 & $1,08^{6}$ & 161,28 & $7,96 \pm 0,79 \mathrm{~b}$ & 75,63 & 2 \\
\hline Lorsban $^{B} 480 \mathrm{BR} /$ clorpirifós $^{7}$ & 1,00 & 0,24 & 169,25 & $0,00 \pm 0,00 \mathrm{c}$ & 100,00 & 4 \\
\hline \multicolumn{7}{|c|}{ Bioensaio III } \\
\hline Água destilada / testemunha negativa & ---- & ----- & 156,37 & 29,29 a & - & - \\
\hline Zapp Qi ${ }^{\circledR} /$ glyphosate & 4,20 & $1,05^{6}$ & 113,34 & $18,60 \mathrm{a}$ & 36,48 & 2 \\
\hline Roundup $^{\mathbb{B}}$ WG / glyphosate & 3,50 & $1,26^{6}$ & 138,46 & $5,08 \mathrm{~b}$ & 82,67 & 3 \\
\hline Finale $^{\circledR} /$ glufosinato - sal de amônio & 1,50 & 0,15 & 135,59 & $1,28 \mathrm{c}$ & 95,64 & 3 \\
\hline Gramoxone $^{\circledR} 200 /$ dicloreto de paraquat & 3,00 & 0,30 & 127,66 & $0,01 \mathrm{~d}$ & 99,95 & 4 \\
\hline Lorsban $^{\circledR} 480$ BR / clorpirifós ${ }^{7}$ & 1,00 & 0,24 & 94,36 & $0,00 \mathrm{e}$ & 100,00 & 4 \\
\hline \multicolumn{7}{|c|}{ Bioensaio IV } \\
\hline Água destilada / testemunha negativa & ---- & ---- & 155,64 & $25,19 \pm 0,84 \mathrm{a}$ & - & - \\
\hline Primóleo $^{\mathbb{B}} /$ atrazine & 6,00 & 1,20 & 160,41 & $24,48 \pm 1,48 \mathrm{a}$ & 2,82 & 1 \\
\hline Gesaprim $^{\mathbb{B}}$ GrDA / atrazine & 3,50 & 1,54 & 161,59 & $17,12 \pm 0,85 \mathrm{~b}$ & 32,03 & 2 \\
\hline Primatop ${ }^{\mathbb{R}} \mathrm{SC} /$ atrazine + simazine & 8,00 & $1,00+1,00$ & 171,87 & $14,93 \pm 1,44 \mathrm{~b}$ & 40,71 & 2 \\
\hline Primestra Gold ${ }^{\mathbb{R}} /$ atrazine + S-metolachlor & 4,50 & $0,83+0,65$ & 169,15 & $0,16 \pm 0,14 \mathrm{c}$ & 99,36 & 4 \\
\hline Lorsban $^{\circledR} 480 \mathrm{BR} /$ clorpirifós $^{7}$ & 1,00 & 0,24 & 164,40 & $0,00 \pm 0,00 \mathrm{c}$ & 100,00 & 4 \\
\hline
\end{tabular}

${ }^{1 /} \mathrm{DC}=$ dosagem do produto comercial $\left(\mathrm{kg}\right.$ ou $\left.\mathrm{L} \mathrm{ha}^{-1}\right)$.

2/ C.i.a. = concentração $(\%)$ do ingrediente ativo na calda utilizada nos bioensaios.

3/ Médias seguidas por letras idênticas não diferem significativamente $(\mathrm{p}>0,05)$ pelo teste de Tukey-Kramer (bioensaio II: $\mathrm{F}=61,30$;.gl=5; $\mathrm{p}=0,0001$; bioensaio IV: $\mathrm{F}=132,71 ; \mathrm{gl}=5 ; \mathrm{p}=0,0001$; bioensaio $\mathrm{V}: \mathrm{F}=78,01 ; \mathrm{gl}=5 ; \mathrm{p}=0,0001$ ) ou pelo teste (Kruskal-Wallis) BonferroniDunn $t$ (bioensaio I: $\mathrm{k}=17,4659 ; \mathrm{p}=0,0037$; bioensaio III: $\mathrm{k}=22,0748 ; \mathrm{p}=0,0005$; bioensaio VI: $\mathrm{k}=19,8162 ; \mathrm{p}=0,0014$ )

${ }^{4} / \mathrm{RP}=$ redução no parasitismo comparado com a testemunha negativa (água destilada) do bioensaio.

5/ Classes da IOBC/WPRS: 1, inócuo (<30\%); 2, levemente nocivo (30-79\%); 3, moderadamente nocivo (80-99\%); e 4, nocivo ( $>99 \%)$.

6/ Concentração (\%) do equivalente ácido na calda utilizada nos bioensaios.

${ }^{7 /}$ Testemunha positiva - inseticida reconhecidamente nocivo pela IOBC/WPRS.

WPRS para T. cacoeciae. Com base nos resultados obtidos no presente trabalho, verificouse que o número de fêmeas de $T$. pretiosum nas gaiolas permitiu a realização e não interferiu na reprodutibilidade dos bioensaios em laboratório.

Na testemunha negativa, o número de ovos parasitados por fêmea de $T$. pretiosum variou de 25, 19 a 32,64 (Tabela 2). De acordo com a metodologia padronizada pela IOBC, a validação dos testes de seletividade está baseada em alguns critérios, sendo um dos mais importantes o número de ovos parasitados por fêmea para o tratamento testemunha negativa. Quanto à espécie $T$. cacoeciae, a metodologia proposta pela IOBC preconiza um mínimo de 15 ovos parasitados por fêmea (Hassan et al., 2000). Para T. pretiosum ainda não há um número mínimo estabelecido para validação de testes de seletividade, porém, de acordo com estudo sobre o efeito de temperatura no 
desenvolvimento de $T$. pretiosum em ovos de A. Kuehniella realizado por Maceda et al. (2003), a $25^{\circ} \mathrm{C}$ as fêmeas parasitaram em média 5,74 ovos por dia. Com base nos resultados de parasitismo de $T$. pretiosum da testemunha negativa dos bioensaios do presente trabalho (Tabela 2), em seis dias, verificaram-se valores próximos aos encontrados por esses autores e quase o dobro daquele estabelecido para T. cacoeciae por Hassan et al. (2000).

Os herbicidas (\% de ingrediente ativo ou equivalente ácido na calda) causaram reduções no parasitismo de $T$. pretiosum, que variou de 2,82 a 99,95\%, predominando os classificados como levemente nocivos (classe 2), como Agrisato $480 \mathrm{SL}$ (1,08\% glyphosate), Gesaprim GrDA (1,54\% atrazine), Glifos ( $0,36 \%$ glyphosate), Glyphosate Nortox (1,08\% glyphosate), Gliz 480 SL (1,08\% glyphosate), Polaris (0,90\% glyphosate), Primatop SC $(1,00 \%$ atrazine $+1,00 \%$ simazine), Sanson 40 SC $(0,03 \%$ nicosulfuron), Trop (1,08\% glyphosate) e Zapp Qi (1,05\% glyphosate), os quais representaram $41,67 \%$ dos herbicidas testados. Já os moderadamente nocivos (classe 3 ) aos adultos de T. pretiosum, como Finale $(0,15 \%$ glufosinato - sal de amônio), Herbadox $(0,88 \%$ pendimethalin), Poast $(0,12 \%$ setoxidim), Roundup Original (1,08\% glyphosate), Roundup Transorb ( $1,08 \%$ glyphosate) e Roundup WG $(1,26 \%$ glyphosate), representaram $25,00 \%$ dos herbicidas testados; somente $8,33 \%$ foram considerados nocivos (classe 4), sendo Gramoxone 200 ( $0,30 \%$ dicloreto de paraquat) e Primestra Gold $(0,83 \%$ atrazine $+0,65 \%$ S-metolachlor $)$ os representantes dessa categoria (Tabela 2, bioensaios I a VI).

A inocuidade (classe 1) dos herbicidas aos adultos de $T$. pretiosum foi verificada somente para Callisto $(0,10 \%$ mesotriona), Equip Plus $(0,02 \%$ foramsulfuron $+0,002 \%$ iodosulfuronmetílico), Extrazin SC (0,85\% atrazine $+0,85 \%$ simazine), Primóleo (1,20\% atrazine), Provence $750 \mathrm{WG}(0,03 \%$ isoxaflutole) e Siptran 500 SC (1,55\% atrazine), que representaram $25,00 \%$ dos herbicidas testados (Tabela 2, bioensaios IV a V).

O herbicida Extrazin SC foi inócuo (classe 1) e Primatop SC foi levemente nocivo (classe 2) aos adultos de $T$. pretiosum, ambos os herbicidas pertencentes ao grupo químico Triazina (ingrediente ativo atrazine + simazine).
Resultados similares foram encontrados por Hassan et al. (1987) quando classificaram Gesatop 50 (i.a. simazine), na concentração de $0,375 \%$ do produto comercial, como inócuo aos adultos de $T$. cacoeciae. $\mathrm{O}$ ingrediente ativo atrazine também apresentou classificações distintas no presente trabalho, sendo classificado como inócuo (Siptran 500 SC) e levemente nocivo (Gesaprim GrDA) (Tabela 2, bioensaio IV e VI). Hassan et al. (1988) também classificaram Gesaprim 50 (0,335\% atrazine) como levemente nocivo (classe 2) aos adultos de $T$. cacoeciae, porém utilizando formulação comercial distinta da empregada no presente trabalho.

Resultados similares para Roundup Original foram obtidos por Manzoni et al. (2006b) e Giolo et al. (2005a) para $T$. pretiosum, porém utilizando 12 e $8 \mathrm{~L} \mathrm{ha}^{-1}$, respectivamente, dosagens estas superiores à usada no presente trabalho: $6 \mathrm{~L} \mathrm{ha}^{-1}$ (Tabela 2, bioensaio I). O herbicida Roundup WG (1,26\% glyphosate) também foi classificado como moderadamente nocivo (classe 3) aos adultos de $T$. pretiosum por Manzoni et al. (2007), ao testarem agrotóxicos registrados para a cultura da maçã. Efeitos deletérios de Glyphosate Nortox (1,08\% glyphosate) e Gliz 480 CS (1,08\% glyphosate) sobre $T$. pretiosum também foram observados pelos autores, porém com toxicidade superior (classe 3) à encontrada no presente trabalho (classe 2) (Tabela 2, bioensaio II) para ambos os herbicidas. Entretanto, Giolo et al. (2005a) classificaram Roundup WG como levemente nocivo (classe 2) aos adultos de $T$. pretiosum ao obterem redução no parasitismo de $61,56 \%$, esta inferior à encontrada no presente trabalho $(82,67 \%)$ (Tabela 2, bioensaio III), embora tenham utilizado dosagem $14,29 \%$ superior do produto comercial. Estes mesmos autores também classificaram Glyphosate Nortox e Gliz 480 CS como moderadamente nocivos (classe 3 ) aos adultos de $T$. pretiosum. Resultados distintos foram observados por Hassan et al. (1988), ao classificarem o herbicida Roundup (0,36\% glyphosate) como levemente nocivo aos adultos de $T$. cacoeciae.

De acordo Giolo et al. (2005a), os herbicidas Zapp Qi e Roundup Transorb, ambos testados na concentração de $14,40 \mathrm{mg}$ de equivalente ácido de glyphosate por litro de água, foram classificados como levemente nocivo 
(classe 2) e moderadamente nocivo (classe 3), respectivamente, aos adultos de $T$. pretiosum. A mesma classificação foi obtida no presente trabalho (Tabela 2, bioensaios III e I), mas utilizando Zapp Qi e Roundup Transorb nas concentrações de 10,50 e 10,80 mg de equivalente ácido de glyphosate por litro de água, respectivamente.

Os demais herbicidas à base de glyphosate - Agrisato 480 SL, Glifos e Trop - foram classificados como levemente nocivos (classe 2) (Tabela 2, bioensaios I e II) aos adultos de T. pretiosum, de maneira semelhante para a maioria dos demais herbicidas com esse ingrediente ativo. Considerando o sal presente nas formulações comerciais à base de glyphosate, verifica-se que a maioria dos produtos com mesma concentração de equivalente ácido e com sal de isopropilamina foi levemente nociva (classe 2) aos adultos de $T$. pretiosum, exceto Roundup Original e Roundup Transorb, que foram moderadamente nocivos (classe 3). Entretanto, Giolo et al. (2005a) verificaram maior nocividade a esse parasitóide por aquelas formulações de glyphosate com sal de isopropilamina, porém os autores utilizaram concentração de 14,40 mg de equivalente ácido de glyphosate por litro de água, uma concentração que foi superior à de todos os herbicidas à base de glyphosate testados no presente trabalho.

Resultados similares aos do presente trabalho também foram observados por Manzoni et al. (2006b) para o herbicida Finale, que foi classificado como moderadamente nocivo (classe 3) ao T. pretiosum. Hassan et al. (1991), entretanto, classificaram o herbicida Basta, na concentração de $0,10 \%$ de glufosinato sal de amônio, como levemente nocivo (classe 2) aos adultos de $T$. cacoeciae, concentração esta $33,33 \%$ inferior à utilizada no presente traba1ho $(0,15 \%)$. Resultados distintos para o ingrediente ativo setoxidim foram encontrados por Hassan et al. (1994), que classificaram Fervinal Plus $(0,105 \%$ setoxidim) como nocivo (classe 4) aos adultos de $T$. cacoeciae.

O herbicida Gramocil (0,20\% paraquat + $0,10 \%$ diuron) foi testado por Bastos et al. (2005), que ofertaram ovos de A. kuehniella e Sitotroga cerealella (Lepidoptera: Gelechiidae) contaminados ao parasitóide $T$. pretiosum, resultando em parasitismos de 53,89 e
$36,02 \%$, respectivamente, ambos diferindo do tratamento testemunha. Trabalhos envolvendo somente o ingrediente ativo paraquat não foram encontrados na literatura, entretanto se verificou alta mortalidade de adultos de T. pretiosum no presente estudo (Tabela 2, bioensaio III), bem como em pesquisas que envolveram outros ingredientes ativos junto ao paraquat (Bastos et al., 2005).

Na literatura não foram encontrados trabalhos relacionados à seletividade de herbicidas a Trichogramma para a cultura do milho, pois somente alguns inseticidas foram testados até o momento (Cruz, 1995; Pratissoli et al., 2004). Dessa forma, verificou-se que testes de seletividade de herbicidas a $T$. pretioum são de fundamental importância para o MIP na cultura do milho, na tentativa de tornar possivel a associação de diferentes métodos de supressão de pragas, como o controle biológico e o controle químico.

De acordo com os resultados obtidos, os seis herbicidas classificados como inócuos (classe 1) - Callisto, Equip Plus, Extrazin SC, Primóleo, Provence 750 WG e Siptran 500 SC - não seguem para testes posteriores, conforme metodologia da IOBC/WPRS, e são seletivos aos adultos de $T$. pretiosum para as dosagens e concentrações testadas. Já os herbicidas classificados como nocivos (classes 2, 3 e 4) Agrisato 480 SL, Finale, Gesaprim GrDA, Glifos, Glyphosate Nortox, Gliz 480 SL, Gramoxone 200, Herbadox, Poast, Polaris, Primatop SC, Primestra Gold, Roundup Original, Roundup Transorb, RoundupWG, Sanson 40 SC, Trop e Zapp Qi -, que representam 75,00\% dos herbicidas testados, deverão passar para as próximas etapas, que envolverão testes em condições de laboratório sobre as fases imaturas do parasitóide $T$. pretiosum e adultos a campo.

Portanto, a utilização de herbicidas seletivos nos primeiros estádios fenológicos da cultura do milho permite o incremento natural das populações de $T$. pretiosum, os quais poderão atuar como agentes de controle biológico no manejo dos insetos-praga. Aliado a isso, o número de aplicações de inseticidas poderá ser minimizado, resultando assim numa agricultura de menor impacto aos agricultores e ao ambiente.

Planta Daninha, Viçosa-MG, v. 26, n. 2, p. 343-351, 2008 


\section{AGRADECIMENTOS}

À CAPES, pela concessão da bolsa de mestrado ao primeiro autor. Ao CNPq e Fapergs, pelo apoio financeiro para a realização desta pesquisa.

\section{LITERATURA CITADA}

BASTOS, C. S.; ALMEIDA, R. P.; SUINAGA, F. A. Selectivity of pesticides used on cotton (Gossypium hirsutum) to Trichogramma pretiosum reared on two laboratory-reared hosts. Pestic. Manag. Sci., v. 62, n. 1, p. 91-98, 2005.

CAÑETE, C. L. Seletividade de inseticidas a espécies de Trichogramma (Hymenoptera: Trichogrammatidae). 2005. 106 f. Tese (Doutorado em Ciências) - Universidade Federal do Paraná, Curitiba, 2005.

CARVALHO, G. A.; PARRA, J. R. P.; BAPTISTA, G. C. Bioatividade de produtos fitossanitários utilizados na cultura do tomateiro (Lycopersicum esculentum Mill.) a Trichogramma pretiosum Riley, 1879 (Hymenoptera: Trichogrammatidae) nas gerações $\mathrm{F}_{1}$ e $\mathrm{F}_{2}$. Ci. Agrotec., v. 27, n. 2 , p. $261-270,2003$

CASTELO BRANCO, M.; FRANÇA, F. H. Impacto de inseticidas e bioinseticidas sobre adultos de Trichogramma pretiosum. Hortic. Bras., v. 13, n. 2, p. 199-201, 1995.

CRUZ, I. A lagarta-do-cartucho na cultura do milho. Sete Lagoas: Embrapa/Centro Nacional de Pesquisa de Milho e Sorgo, 1995. 45 p. (Circular Técnica, 21).

GIOLO, F. P.et al. Seletividade de formulações de glyphosate a Trichogramma pretiosum (Hymenoptera: Trichogrammatidae). Planta Daninha, v. 23, n. 3, p. 457$462,2005 a$

GIOLO, F. P. et al. Seletividade de agrotóxicos indicados na produção integrada de pêssego a Trichogramma pretiosum Riley, 1879 (Hymenoptera: Trichogrammatidae). R. Bras. Frutic., v. 27, n. 2, p. 222-225, 2005b

HASSAN, S. A. Guideline for the evaluation of side effects of plant protection products on Trichogramma cacoeciae Marchal (Hym., Trichogrammatidae). IOBC/WPRS Bull., v. 21, n. 6, p. 119-128, 1998.

HASSAN, S. A.; ABDELGADER, H. A sequential testing program to assess the effects of pesticides on Trichogramma cacoeciae Marchal (Hym., Trichogrammatidae). IOBC/WPRS Bull., v. 24, n. 4, p. 7181,2001
HASSAN, S. A. et al. Results of the third joint pesticide testing programme by the IOBC/WPRS - Working Group "Pesticides and Beneficial Organisms". Zeitschrift Angewandte Entomol., v. 103, p. 92-107, 1987.

HASSAN, S. A. et al. Results of the fourth joint pesticide testing programme carried out by the IOBC/WPRS Working Group "Pesticides and Beneficial Organisms". Zeitschrift Angewandte Entomol., v. 105, p. 321-329, 1988.

HASSAN, S. A. et al. Results of the fifth joint pesticide testing programme carried out by the IOBC/WPRS Working Group "Pesticides and Beneficial Organisms". Entomophaga, v. 36, n. 1, p. 55-67, 1991

HASSAN, S. A. et al. Results of the sixth joint pesticide testing programme carried out by the IOBC/WPRS Working Group "Pesticides and Beneficial Organisms". Entomophaga, v. 39, n. 1, p. 107-119, 1994.

HASSAN, S. A. et al. A laboratory method to evaluate the side effects of plant protection products on Trichogramma cacoeciae Marchal (Hym., Trichogrammatidae). In: CANDOLFI, M.P. (Eds.). Guidelines to evaluate sideeffects of plant protection products to non-target arthropods. Reinheim: IOBC/WPRS, 2000. p. 107-119.

HOHMANN, C. L. Efeito de alguns inseticidas sobre adultos de Trichogramma pretiosum Riley. An. Soc. Entomol. Brasil, v. 22, n. 3, p. 563-568, 1993.

\section{INSTITUTO BRASILEIRO DE GEOGRAFIA E} ESTATÍSTICA - IBGE. Levantamento sistemático da produção agrícola. Disponível em: (http://www.ibge.gov.br.) Acesso em: 6 de jan. de 2007.

MACEDA, A.; HOHMANN, C. L.; SANTOS, H. R. Temperature effects on Trichogramma pretiosum Riley and Trichogrammatoidea annulata De Santis. Braz. Arch. Biol. Technol., v. 46, n. 1, p. 27-32, 2003.

MAGALHÃES, P. C. et al. Eficiência dos dessecantes paraquat e diquat na qualidade fisiológica de sementes de milho. Sete Lagoas: Embrapa/Centro Nacional de Pesquisa de Milho e Sorgo, 2005. 4 p. (Comunicado Técnico, 123)

MANZONI, C. G. et al. Susceptibilidade de adultos de Trichogramma pretiosum Riley (Hymenoptera: Trichogrammatidae) a fungicidas utilizados no controle de doenças da macieira. Neotr. Entomol., v. 35, n. 2, p. 223 $230,2006 a$.

MANZONI, C. G. et al. Seletividade de agrotóxicos usados na produção integrada de maçã para adultos de Trichogramma pretiosum. Pesq. Agropec. Bras., v. 41, n. 10 , p. 1461-1467, 2006b. 
MANZONI, C. G. et al. Seletividade de agroquímicos utilizados na produção integrada de maçã aos parasitóides Trichogramma pretiosum Riley e Trichogramma atopovirilia Oatman \& Platner (Hymenoptera: Trichogrammatidae). Bioassay, v. 2, n. 1, 2007. Disponível em (www.seb.org.br/bioassay) Acesso em 03 de mar. de 2007.

MORANDI FILHO, W. J. et al. Ação de produtos naturais sobre a sobrevivência de Argyrotaenia sphaleropa (Meyrick) (Lepidoptera: Tortricidae) e seletividade de inseticidas utilizados na produção orgânica de videira sobre Trichogramma pretiosum Riley (Hymenoptera: Trichogrammatidae). Ci. Rural, v. 36, n. 4, p. 1072-1078, 2006.

MOURA, A. P. et al. Selectivity evaluation of insecticides used to control tomato pests to Trichogramma pretiosum. BioControl, v. 51, n. 6, p. 769-778, 2006.

PRATISSOLI, D. et al. Ação transovariana de lufenuron (50 G/L) sobre adultos de Spodoptera frugiperda (J.E. Smith) (Lepidoptera: Noctuidae) e seu efeito sobre o parasitóide de ovos Trichogramma pretiosum Riley (Hymenoptera: Trichogrammatidae). Ci.. Agrotec., v. 28, n. 1, p. 9-14, 2004.
SÁ, L. A. N.; PARRA, J. R. P. Efeito do número e intervalo entre liberações de Trichogramma pretiosum Riley no parasitismo e controle de Helicoverpa zea (Boddie), em milho. Sci. Agric., v. 50, n. 3, p. 355-359, 1993.

SAS LEARNING EDITION. Getting Started with the SAS Learning Edition. Statistical Analysis System. Cary: SAS Institute, 2002. 2 CD-ROM.

SILVA, A. A. et al. Interação entre herbicida e inseticida sobre o milho-pipoca (Zea mays), as plantas daninhas e a lagarta-do-cartucho. Planta Daninha, v. 25, n. 1, p. 181186, 2007.

VIÑUELA, E. Efectos secundarios de los plaguicidas en los enemigos naturales - Importancia para la producción integrada de fruta. In: SEMINÁRIO BRASILEIRO DE PRODUÇÃO INTEGRADA DE FRUTAS, 4., 2002, Bento Gonçalves. Anais... Bento Gonçalves: Embrapa Uva e Vinho, 2002. p. $17-40$.

ZHANG, W.; HASSAN, S. A. Rationalising the standard method to test the side-effects of pesticides on

Trichogramma cacoeciae, reducing the number of parasitoids tested. IOBC/WPRS Bull., v. 23, p. 49-53, 2000. 